\title{
Taste Response Variability and Temporal Coding in the Nucleus of the Solitary Tract of the Rat
}

\author{
Patricia M. Di Lorenzo ${ }^{1}$ and Jonathan D. Victor ${ }^{2}$ \\ ${ }^{1}$ Department of Psychology, State University of New York, Binghamton 13902-6000; and ${ }^{2}$ Department of Neurology and Neuroscience, \\ Weill Medical College of Cornell University, New York City, New York 10021
}

Submitted 25 February 2003; accepted in final form 25 April 2003

Di Lorenzo, Patricia M. and Jonathan D. Victor. Taste response variability and temporal coding in the nucleus of the solitary tract of the rat. J Neurophysiol 90: 1418-1431, 2003. 10.1152/jn.00177.2002. Theories of taste coding in the brain stem have been based on the idea that taste responses are integrated over time without regard to the temporal structure of the taste-evoked spike train. In the present experiment, the reliability of response rate across stimulus repetitions and the potential contribution of temporal coding to the discrimination of taste stimuli was examined. Taste stimuli representing the four basic taste qualities were presented repeatedly, and electrophysiological responses were recorded from single cells in the nucleus of the solitary tract (NTS) of anesthetized rats. Blocks of the four tastants were repeated for as long as the cell remained isolated. Nineteen cells were recorded with between 8 and 27 repetitions of each stimulus. Response magnitude to a given tastant varied widely within some NTS cells. This impacted the determination of both the breadth of tuning and best stimulus for a given cell. The contribution of spike timing and the pattern of interspike intervals to discrimination of taste stimuli was evaluated by an information-theoretic approach based on two families of metrics. Spike timing significantly contributed to the discrimination of taste qualities in 10 of 19 (53\%) cells. This contribution was especially notable during the initial $2 \mathrm{~s}$ of the response. Those cells that showed the most variable firing rates in response to repetition of taste stimuli tended to show the largest contribution of temporal coding. These results suggest that, in addition to response rate, the temporal parameters of responses may convey information about taste stimuli in the NTS.

\section{N T R O D U C T I O N}

The nature of the neural representation of gustatory quality has been a matter of debate for more than half a century. In that time, two major theories have emerged that have dominated the literature. These are the labeled-line theory (e.g., Frank 1973, 2000; Lundy and Contreras 1999; Scott and Giza 1990) and the across-neuron-pattern theory (e.g., Doetsch and Erickson 1970; Ganchrow and Erickson 1970; Yamamoto and Yuyama 1987). Because taste-responsive cells in the nervous system are almost always multisensitive across taste qualities, the across-neuronpattern theory argues that the pattern of response across cells is the critical feature for identification of and comparison across tastants of different qualities (e.g., sweet, sour, salty, or bitter). Not inconsistent with this idea is the labeled-line theory, which suggests that stimuli of a given taste quality are encoded by those cells that respond preferentially to that stimulus. Both of

Address for reprint requests: Dept. of Psychology, Box 6000, SUNY at Binghamton, Binghamton, NY 13902-6000 (E-mail: diloren@binghamton.edu). these theories are essentially spatial theories based on the assumption that neural responses are integrated over time. That is, the basic unit of data for both theories is a measure of the neural firing rate over an (arbitrary) interval of time. This measure ignores any potentially systematic variations in the temporal patterns of response that might convey information about a taste stimulus (see Katz et al. 2002 for a review).

In other sensory systems, several authors have emphasized the role of response dynamics, either from the point of view of its origin in neural circuitry (especially in vision (DeAngelis et al. 1995; Ringach et al. 1997; Shapley and Enroth-Cugell 1984; Victor 1999) or its potential role in temporal coding (Cariani 1995; Eggermont 1998; Hopfield 1995; Laurent 1999; McClurkin et al. 1991)

In the field of gustation, several investigators have attempted to assess the relationship between the temporal pattern of taste-evoked spike trains and the stimuli that produced them. These efforts have focused on both peripheral nerve fibers (Bradley et al. 1983; Fishman 1957; Mistretta 1972; Nagai and Ueda 1981; Ogawa et al. 1973, 1974) and on neurons in the CNS (Covey 1980; Di Lorenzo and Schwartzbaum 1982; Funakoshi and Ninomiya 1977; Nuding et al. 1991; Scott and Erickson 1971; Scott and Perrotto 1980; Travers and Norgren 1989; Yamamoto et al. 1984). In general, these reports have suggested that temporal patterns of response across neurons do not convey enough information to unambiguously identify a taste stimulus (see, for example, Di Lorenzo and Schwartzbaum 1982; Nagai and Ueda 1981). However, some investigators have suggested that temporal coding may be used by some cells and not others (Bradley et al. 1983; Di Lorenzo and Schwartzbaum 1982; Nagai and Ueda 1981).

There have been several issues that have limited investigations of temporal coding in gustation. One is the issue of response variability. That is, almost without exception, studies of taste coding have relied on the analysis of responses to single presentations of taste stimuli. When responses to multiple presentations of the same stimulus are recorded, the stability of the response is almost never explicitly examined (for an exception see Ogawa et al. 1973, 1974). Obviously, this makes any conclusions about temporal patterns of responses problematical. Another issue concerns the definition of temporal coding and, by extension, the techniques used to assess temporal coding. For example, temporal coding may be defined as unique distributions of interspike intervals (ISIs) associated with different taste qualities (e.g., Nagai and Ueda 1981; Nuding et al. 1991) and/or as a characteristic time course of 
firing rate evoked by different taste qualities (e.g., Di Lorenzo and Schwartzbaum 1982). Because the reciprocal of an ISI estimates the instantaneous firing rate, it might at first appear that rate coding and coding by ISI pattern are indistinguishable. However, this is not the case. For example, altering a spike train by changing an ISI changes the time of all subsequent spikes and thus shifts the entire downstream estimate of rate. Thus two spike trains that have only a single mismatched ISI may correspond to very different rate functions. Another kind of distinction relies on the observation of an ensemble of responses to repeated presentations of several stimuli. Such response sets might be distinguishable on the basis of consistently different rates even though they had variable and largely overlapping ISIs (as would be expected from Poisson-like firing). Alternatively, they might have the same rates, but different distributions of ISIs (e.g., one stimulus elicits a clocklike response, whereas the other stimulus elicits a Poisson-like response of the same mean firing rate). Thus with an appropriate analytical method and access to many replicate runs, rate coding and interval coding can be distinguished.

The present experiment was designed with two purposes in mind. First, the variability of taste responses over repeated presentations was assessed. The potential for such variability to affect the order of effectiveness of taste stimuli within a neuron was also examined. Second, the extent to which the temporal parameters of taste responses can be used to distinguish among taste qualities was assessed using quantitative methods first proposed by Victor and Purpura (1996). These analytical techniques are designed to segregate the contributions of firing rate, spike timing, and the sequence of ISIs to the discrimination of taste quality. This type of analysis has been used successfully to identify the contribution of temporal coding in the visual system (Reich et al. 2001; Victor and Purpura 1996). This approach also is sensitive to response variability in that it relies on a comparison of the similarity of responses to repeated presentations of the same stimulus to the similarity of responses to presentations of different stimuli.

\section{METHODS}

\section{Subjects}

Eighteen male Sprague-Dawley rats (325-475 g) served as subjects. Animals were housed individually in stainless steel cages and maintained on a 12-hr light/dark schedule (lights on at 7:00 a.m.). Food and water were available ad libitum.

\section{Surgery}

All animals were fully anesthetized with urethan $(1.5 \mathrm{gm} / \mathrm{kg}$ ip administered in 2 equal doses $20 \mathrm{~min}$ apart) prior to surgical manipulation. Rats were tracheotomized, and an endotracheal tube was inserted to facilitate breathing. Animals were then mounted in a stereotaxic instrument with the nose positioned $5.5 \mathrm{~mm}$ below the interaural line. This allowed vertical access to the nucleus of the solitary tract (NTS) without disturbing any major blood vessels or sinuses. The scalp was incised, and the skin and fascia were retracted to expose the dorsal surface of the skull. Next the occipital bone was removed, and the uvular and nodular portions of the cerebellum were gently aspirated to expose the surface of the medulla. The cavity was filled with warm mineral oil to prevent dehydration. A nontraumatic head holder was positioned above six stainless steel screws embedded in the skull, and the entire assembly was embedded in dental acrylic cement. This permitted the ear and tooth bars to be removed to allow access to the mouth while preserving the correct orientation of the head. Core temperature was maintained at $37^{\circ} \mathrm{C}$ with an anal thermister probe connected to a heating pad.

\section{Recording}

Extracellular recordings were made from single cells in the NTS with etched tungsten microelectrodes, insulated except for the tip (FHC, 18-20 M, $1 \mathrm{~V}$ at $1 \mathrm{kHz}$ ). Electrodes were lowered into the medulla with a microdrive. Previous work in our lab has shown that the NTS is located $2.7 \mathrm{~mm}$ rostral and $1.8 \mathrm{~mm}$ lateral to the obex and 0.8-1.2 mm below the surface of the medulla. When the electrode was located at $\sim 200 \mu \mathrm{m}$ above the NTS, each cell that was isolated as the electrode was advanced was tested for taste responsivity with all four taste stimuli, each followed by a distilled water rinse. Electrophysiological responses to the various taste stimuli were recorded on VHS tape with a PCM instrumentation recorder (Vetter).

Waveforms associated with single cells were isolated using the Discovery software package (DataWave). Briefly, waveforms were screened initially by amplitude above the noise level. Next, eight attributes of each waveform, e.g., peak to peak amplitude, width of waveform, etc., were measured, and each pair of attributes was plotted against each other. Waveforms originating from the same cell produced tight clusters of points in one or more of these plots. Because the signal-to-noise ratio was the initial defining feature of the waveform, clusters associated with a given cell appeared in several of these plots. The software permitted the identification of the area of each plot that defined a consistent waveform that could be classified as a single cell. The isolation of each cell was monitored continuously both during the recording session and during the off line analysis to ensure that the signal-to-noise ratio was sufficient and that no other cell entered the record. During a stimulus trial, the occurrence of each spike was identified by the time (to the nearest millisecond) from the initiation of the trial.

\section{Taste stimuli}

Taste stimuli consisted of $\mathrm{NaCl}(\mathrm{N} ; 0.1 \mathrm{M})$, sucrose $(\mathrm{S} ; 0.5 \mathrm{M})$, quinine $\mathrm{HCl}(\mathrm{Q} ; 0.01 \mathrm{M})$, and $\mathrm{HCl}(\mathrm{H} ; 0.01 \mathrm{M})$ dissolved in distilled water. All tastants were made with reagent grade chemicals and presented at room temperature. These concentrations were selected because they evoked approximately half maximal responses from the chorda tympani nerve (Ganchrow and Erickson 1970; Ogawa et al. 1974).

Stimuli were delivered to the oropharyngeal area by a custom-built stimulus-delivery system. Each tastant was presented through an individual stainless steel tube that was perforated along the portion that was located inside the oral cavity. Because the reservoirs containing each taste stimulus were pressurized with compressed air, the release of that pressure through the activation of computer-controlled solenoids resulted in a rapid gush of stimulus that flooded the entire mouth almost immediately. The time from the signal to open the solenoids from the computer until the time that the stimulus contacted the tongue was calibrated as $63 \mathrm{~ms}$ with a range of no more than \pm 5 $\mathrm{ms}$. The flow rate for this system was $5 \mathrm{ml} / \mathrm{s}$.

\section{Testing}

Once a single taste-responsive NTS cell had been identified, testing was begun. Each stimulus was presented in individual trials in the following order: N, S, Q, H. Each trial consisted of a 10-s baseline period where no stimulus was presented, a 5-s stimulus presentation, a 5-s wait, and a 20-s distilled water rinse. Interstimulus interval was 1.5 min. Each block of four tastants, always beginning with $\mathrm{NaCl}$, was repeated for as long as the cell was well isolated. 


\section{Analysis of general response characteristics}

Taste responses were defined as the average firing rate that occurred during the stimulus presentation minus the spontaneous firing rate (defined by average firing rate during the 10-s baseline period for each trial). Responses were considered significant if the response rate was 2.54 SD above or below the baseline firing rate. In the present data set, there were no significant responses where the response rate was below the baseline firing rate.

Cells were classified according to their "best stimulus," i.e., the stimulus that evoked the greatest response, in several ways. First, the best stimulus was determined by the first block of four stimuli that were presented. This is consistent with what would be done in most similar experiments in the literature. Second, the best stimulus was determined by stimulus that evoked the greatest average response magnitude across all repetitions. Third, the best stimulus was determined by the largest response to any stimulus in any presentation. Finally, the best stimulus was determined for each successive block of four tastants. The proportion of blocks where a given stimulus was the best stimulus was also calculated.

To determine the breadth of tuning of a given cell, an uncertainty measure was used (Smith and Travers, 1979). The formula for uncertainty is as follows

$$
U=-1.66\left(\sum_{i=1}^{4} P_{i} \log P_{i}\right)
$$

where $P_{i}$ represents the response to each stimulus expressed as a proportion of the total response to $n$ stimuli. Values close to zero indicate narrow tuning, i.e., the cell responds only to one or two taste stimuli; values close to 1.0 indicate broad tuning, i.e., the cell responds nearly equally to all taste stimuli. The uncertainty measure was calculated for each successive block of stimulus presentations.

To assess the variability of the responses across repetitions, the SD of each response was calculated, along with a coefficient of variation (SD/mean).

\section{Analysis of temporal patterns of response}

To characterize the temporal structure of the responses, spike trains were analyzed by the metric space method of Victor and Purpura (1997). This approach provides a rigorous way to determine whether the statistics of the precise times of individual spikes, or of the pattern of interspike intervals, have the potential to carry information concerning the taste stimuli. The analysis is framed in terms of a family of metrics; i.e., notions of distance (i.e., dissimilarity) between spike trains. The description below is a summary of the procedure; further details can be found in Victor and Purpura (1996, 1997).

The simplest metric of similarity between two responses is equivalent to comparing the number of spikes. This metric is called $D^{\text {count }}$. The distance between two spike trains is measured by the "cost" of changing one response into the other. In this case, each spike that is removed or added incurs a cost of 1 , and no cost is incurred by moving a spike in time. This formalizes the notion that only the spike count, and not the temporal parameters of the responses, are relevant to the "message" conveyed by a spike train. $D^{\text {count }}$ then is identical to the arithmetic difference in the number of spikes in each of the two responses. So, the information conveyed by $D^{\text {count }}$ is that which is conveyed strictly by rate coding without regard to the arrangement of the spikes in time.

The parametric family of metrics denoted $D^{\text {spike }}[q]$ tests the notion that spike times can carry information. In the metric $D^{\text {spike }}[q]$, the distance between two spike trains is again the minimum total cost to transform one spike train into the other, but in contrast to $D^{\text {count }}$, there is now a cost incurred by moving a spike in time. The cost of inserting a spike or deleting it altogether is set at 1 . So the cost of transforming one spike train to another will be at least the difference in the number of spikes between them. In addition, the cost of moving a spike by an amount of time $t$ is set at $q t$, where $q$ is in units of $1 / \mathrm{s}$. If $q$ was set at zero, i.e., there was no cost to moving any spike in time, then the distance (cost) between the two trains would simply be the difference in the number of spikes. Thus $D^{\text {spike}}[0]$ is equivalent to $D^{\text {count }}$. For nonzero values of $q$, the distance (dissimilarity) between two spike trains (in the context of $D^{\text {spike }}[q]$ ) depends not only on the difference in the number of spikes but also on the whether they have a similar pattern in time.

The parameter $q$ can be thought of in two ways. First, it represents the cost of moving a spike $t$ seconds in relation to the cost of adding or deleting a spike altogether. Consider an example of two spike trains, $\mathrm{A}$ and $\mathrm{B}$, that are identical in the number of spikes in each train and in the timing of the spikes in each train except that one spike in $\mathrm{B}$ is displaced by $0.25 \mathrm{~s}$ compared with its counterpart in A (Fig. 1). In that case, the distance between $\mathrm{A}$ and $\mathrm{B}$ (i.e., the cost of making $\mathrm{A}$ identical to B) would vary depending on $q$. If $q$ was 2 , then moving the stray spike in B to match its counterpart in A by $0.25 \mathrm{sec}$ would cost 0.5 ; if $q$ was 4 , the cost would be 1 (the same as deleting a spike altogether), and if $q$ was 8 , the cost would be 2.0. But if $q$ was greater than 8 , then the distance between A and B would still be only 2.0 because the "cheapest" way of transforming A into B would not be to move the spike (which would cost $0.25 q$ ) but would be to delete the stray spike in A and then reinsert it in B (which would cost 2.0). Thus a second meaning of $q$ is in terms of temporal precision. Spikes that are further than $2 / q$ from each other are considered (by this metric) to be unrelated. That is, the dissimilarity between two trains that differ by a single spike displaced by $2 / q$ or more is the same as if the two spikes were arbitrarily far apart. Conversely, if corresponding spikes in two spike trains are less than $2 / q$ from each other, then they are seen as related, i.e., they contribute to the similarity of the spike trains. Equivalently, shifting a spike time by $1 / q$ is considered (by this metric) to make just as much of a difference as deleting the spike altogether. Thus if we define the temporal precision of coding as the difference in the timing of the occurrence of two spikes that makes just as much of a difference to the nervous system as a deletion of a spike, then $1 / q$ is the measure of temporal precision. Thus spike trains are considered similar (in the sense of $D^{\text {spike }}[q]$ ) only if they have approximately the same number of spikes, and these spikes occur at approximately the same times, i.e., within $1 / q$ or less.

The parametric family of metrics denoted by $D^{\text {interval }}[q]$ uses an analogous strategy to test the notion that interspike intervals can carry information irrespective of their timing. In the metric $D^{\text {interval }}[q]$, the

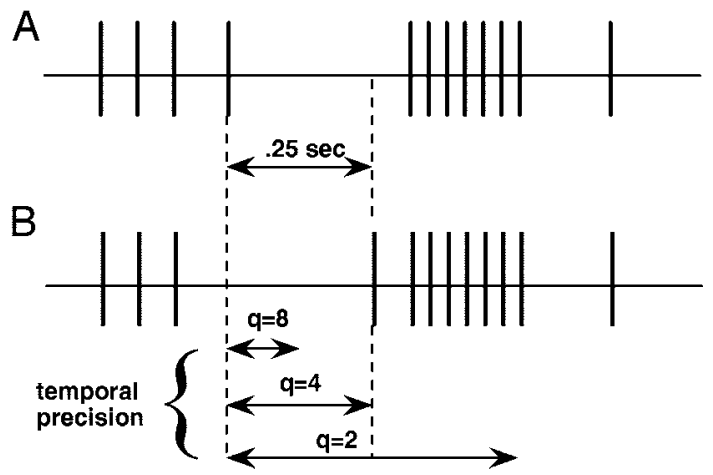

FIG. 1. Example of hypothetical spike trains that illustrate the meaning of $q$. $A$ and $B$ : spike trains that differ only in the timing of 1 spike, which is moved by $0.25 \mathrm{~s}$. As illustrated, the maximum distance that a spike can be moved at a cost that does not exceed the cost of deleting it altogether is $1 / q$. Thus at values of $q$ that are $\geq 4$, the cost of moving a spike by $0.25 \mathrm{~s}$ in time is equal to, or greater than, the cost of deleting the spike altogether. That is, the temporal precision corresponding to $q=4$ is $1 / q$, or $0.25 \mathrm{~s}$. At this level of temporal precision, these spike trains are similar. See text for additional explanation. 
distance between two spike trains is defined as the minimum total cost to transform one spike train into the other via any sequence of insertions of spikes, deletions of spikes, and expansions or contractions of interspike intervals. Note that, for the metric $D^{\text {interval }}[q]$, the intervals, not the spikes, are considered the fundamental unit. Thus even though inserting an interval or stretching an interval may cause the absolute time of some subsequent spikes extend beyond the original analysis interval, the entire set of spikes (considered as a train of the intervals they delineate) enters into the analysis. The cost of changing an interspike interval by an amount $t$ is set at $q t$, and the cost of inserting or deleting a spike is set at 1 . The temporal precision, here defined as the change in an interval of time between spikes that is equivalent in significance to the gain or loss of a spike altogether, is indexed by $1 / q$, as in $D^{\text {spike }}[q]$.

Each metric (i.e., $D^{\text {spike }}[q]$ and $D^{\text {interval }}[q]$, for values of $q$ spaced over a range of interest), is then evaluated by how faithfully they classify the observed responses to the four taste stimuli. Classification will be good if the distance between different responses to the same tastant is usually smaller than the distance between responses to different tastants. Because there are four equally likely tastants, perfect classification corresponds to an information $H=2$ bits $\left(\log _{2} 4=\right.$ 2 ). Conversely, if the notion of distance (or similarity) associated with a metric has little to do with whether or not the spike trains are elicited by the same tastant, then classification will be nearly random. In the extreme, this corresponds to an information $H=0$ bits per response.

For limitingly small values of $q, D^{\text {spike }}[q]$ or $D^{\text {interval }}[q]$ both become independent of the temporal arrangement of spikes because moving a spike in time, or stretching an interval, has no effect on distance. Thus the value of the information associated with $D^{\text {spike }}[0]$ or $D^{\text {interval }}[0]$ measures the extent to which responses to the tastants can be distinguished based solely on their spike counts. As $q$ increases, an increase in $H$ for $D^{\text {spike }}[q]$ (or $D^{\text {interval }}[q]$ ) indicates that consideration of spike times (or spike intervals) allow for a more reliable classification of the responses. When such an increase is observed (e.g., Figs. 6-8), we anticipate that estimated information will eventually decrease again when $q$ exceeds some value $q_{\max }$. This subsequent decrease indicates that temporal structure is only useful for classifying stimuli up to a certain precision (corresponding to $\left.1 / q_{\max }\right)$. That is, candidate metrics for higher $q$ values, which are sensitive to temporal structure at an even finer temporal scale, are influenced by details of the spike train that are not consistent among the replicate responses to same stimulus and hence are irrelevant to coding.

We performed two kinds of computational analyses to assess the significance of the results of the metric space method. First, values of $H$ calculated by classifying the observed responses were compared with values $H_{0}$ obtained from 10 to 40 surrogate data sets in which the tastants associated with each response were randomly scrambled. This control is necessary because estimates of $H$ have an upward bias, which is conservatively estimated by $H_{0}$. Only values of $H$ that exceed the range (mean $+2 \mathrm{SD}$ ) of values of $H_{0}$ can be considered to represent better-than-chance classification.

In a second analysis, known as "exchange resampling" (Victor and Purpura 1996), we created surrogate data sets that matched the poststimulus histograms of the observed responses and also had the same number of spikes in each response. We then compared values of $H$ obtained from the real data with values $H_{\text {res }}$ obtained from the same analysis on 10-40 of these resampled datasets. If $H$ was not within the range (mean $\pm 2 \mathrm{SD}$ ) of values of $H_{\text {res }}$, we can conclude that the observed temporal coding is not merely due to the average temporal profile of the response to each tastant (with the overall variability in spike count taken into consideration) and that the arrangement of spikes in time in individual trials must play a role.

\section{RE S U L T S}

Electrophysiological responses to taste stimuli were recorded in 19 cells with between 8 and 27 repetitions of each stimulus. In one animal, two cells were isolated from the same electrode. The mean spontaneous and evoked response rates for all cells are shown in Table 1. The order of effectiveness of taste stimuli tested was $\mathrm{NaCl}>\mathrm{HCl}>$ quinine $>$ sucrose. Across cells, the average spontaneous rate was $2.4 \pm 0.6$ (SE) spikes/s (sps). A summary of some additional general response characteristics of the sample are presented in Table 2. As can be seen from this table, $\mathrm{NaCl}$ evoked responses in nearly all NTS cells tested, whereas sucrose responses were evoked in only about a third of the cells. The great majority of cells were broadly tuned across taste stimuli with $68 \%$ responsive to three or four of the tastants tested.

TABLE 1. Mean spontaneous and evoked response rates (sps)

\begin{tabular}{|c|c|c|c|c|c|c|c|c|c|c|c|}
\hline \multirow{2}{*}{$\frac{\text { Cell }}{1}$} & \multicolumn{2}{|c|}{ Spontaneous } & \multicolumn{2}{|c|}{ Sucrose } & \multicolumn{2}{|c|}{$\mathrm{NaCl}$} & \multicolumn{2}{|c|}{$\mathrm{HCl}$} & \multicolumn{2}{|c|}{ Quinine } & \multirow{2}{*}{$\frac{\text { No. Blocks }}{25}$} \\
\hline & 7.2 & 3.5 & -1.8 & 7.4 & 56.6 & 9.4 & 18.2 & 4.4 & 10.3 & 3.1 & \\
\hline 2 & 0.9 & 0.4 & 0.4 & 0.7 & 6.4 & 2.6 & 5.7 & 2.2 & 3.2 & 1.1 & 24 \\
\hline 3 & 0.7 & 0.4 & 0.3 & 0.5 & 4.0 & 1.1 & 8.2 & 3.2 & 1.9 & 0.9 & 17 \\
\hline 4 & 0.3 & 0.2 & 0.2 & 0.4 & 1.9 & 0.9 & 3.0 & 2.1 & 0.9 & 0.5 & 17 \\
\hline 5 & 0.5 & 0.4 & 0.9 & 0.7 & 11.3 & 3.0 & 4.5 & 1.6 & 2.1 & 1.3 & 27 \\
\hline 6 & 0.3 & 0.2 & 0.6 & 0.5 & 3.1 & 1.0 & 3.6 & 0.9 & 1.3 & 0.5 & 25 \\
\hline 7 & 0.8 & 1 & 14.3 & 3.3 & 3.8 & 1.3 & 1.8 & 1.0 & -0.9 & 1.0 & 11 \\
\hline 8 & 1.6 & 1.2 & 0.9 & 0.8 & 5.8 & 0.8 & 1.5 & 0.7 & 1.2 & 0.8 & 25 \\
\hline 9 & 1.1 & 0.5 & 0.3 & 0.8 & 9.8 & 1.8 & 14.9 & 1.7 & 5.1 & 1.4 & 23 \\
\hline 10 & 3.7 & 2.7 & 2.5 & 2.2 & 63.5 & 13.0 & 33.2 & 4.4 & 19.8 & 2.6 & 25 \\
\hline 11 & 5.3 & 2.3 & 0.8 & 2.3 & 16.6 & 3.5 & 12.5 & 2.9 & 8.9 & 2.2 & 16 \\
\hline 12 & 2 & 1 & -0.1 & 0.7 & 11.7 & 2.7 & 5.6 & 1.5 & 4.3 & 1.1 & 18 \\
\hline 13 & 1.4 & 0.6 & 16.7 & 1.4 & 12.7 & 1.2 & 4.9 & 0.8 & 1.9 & 1.0 & 24 \\
\hline 14 & 1.8 & 1 & 17.4 & 2.9 & 10.2 & 1.7 & 5.6 & 1.4 & 2.1 & 2.5 & 14 \\
\hline 15 & 1.9 & 0.5 & 1.0 & 1.2 & 16.2 & 1.9 & 14.1 & 3.4 & 8.6 & 1.3 & 13 \\
\hline 16 & 8.8 & 5.2 & 5.6 & 5.1 & 34.8 & 14.3 & 14.2 & 8.3 & 12.5 & 5.3 & 8 \\
\hline 17 & 2.4 & 0.8 & 10.7 & 2.3 & 29.3 & 4.8 & 17.8 & 2.1 & 10.8 & 1.9 & 25 \\
\hline 19 & 0.4 & 0.3 & 9.3 & 2.3 & 2.5 & 1.3 & 2.1 & 0.9 & 1.2 & 1.1 & 21 \\
\hline 22 & 4.3 & 1.3 & 1.4 & 2.3 & 40.7 & 8.2 & 34.9 & 6.6 & 15.5 & 4.2 & 19 \\
\hline Overall & 2.4 & 2.4 & 4.3 & 1.4 & 18.0 & 4.2 & 10.9 & 2.3 & 5.8 & 1.3 & \\
\hline
\end{tabular}

Values are means $\pm \mathrm{SD}$. 
TABLE 2. General response characteristics

\begin{tabular}{lcc}
\hline \hline \multicolumn{2}{c}{ Significant Responses } \\
\hline Stimulus & Responses & Percent Total Cells \\
\hline Sucrose & 6 & 31 \\
$\mathrm{NaCl}$ & 18 & 95 \\
$\mathrm{HCl}$ & 15 & 79 \\
Quinine & 11 & 59 \\
& & \\
& Breadth of Tuning & \\
& Stimulus Responses & Cells \\
\hline & (Based on Average Response) & 2 \\
& 4 & 3 \\
& 2 & 3 \\
\hline
\end{tabular}

Variability with stimulus repetition

Taste responses in NTS cells varied in magnitude across blocks of stimulus presentations, sometimes to a large extent. As a result, some characteristics normally used to categorize taste-responsive cells differed over the course of several stimulus repetitions.

For example, the availability of many repetitions of each stimulus offered the opportunity to define the best stimulus of a cell in different ways. The outcome of each of these methods applied to the same cell sometimes differed. The best stimulus for a given cell was determined in three ways: First, the best stimulus was defined as the stimulus that produced the largest response in the first block of trials. This represents the most common method used in most investigations of taste-responsive neurons. Second, the best stimulus was defined as the stimulus that evoked the largest average response across all stimulus repetitions. In 4 of 19 cells (21\%), the best stimulus using the average responses was different than that using the responses from the initial trial. A third method defined the best stimulus as the stimulus that evoked the largest response over all stimulus trials. In all cases, this determination matched that using the average response.

The proportion of blocks of trials where a given cell showed a particular best stimulus was also noted. In 12 of 19 cells $(63 \%)$, the best stimulus remained constant over all blocks of trials tested. This group could be divided into two subgroups: the first of these is illustrated in Fig. 2. This figure shows two examples of the subgroup of five cells where the responses to all stimuli tested were consistently different from each other in every block of trials. Figure 3 shows two examples from the subgroup of the remaining seven cells where, although the best stimulus is clear and reliable across blocks of trials, there is considerable overlap among response magnitudes evoked by the remaining, non-best stimuli. In 7 of 19 (37\%) cells, the best stimulus varied between $\mathrm{NaCl}$ and $\mathrm{HCl}$ across blocks of trials. Figure 4 shows two examples of these cells. The examples

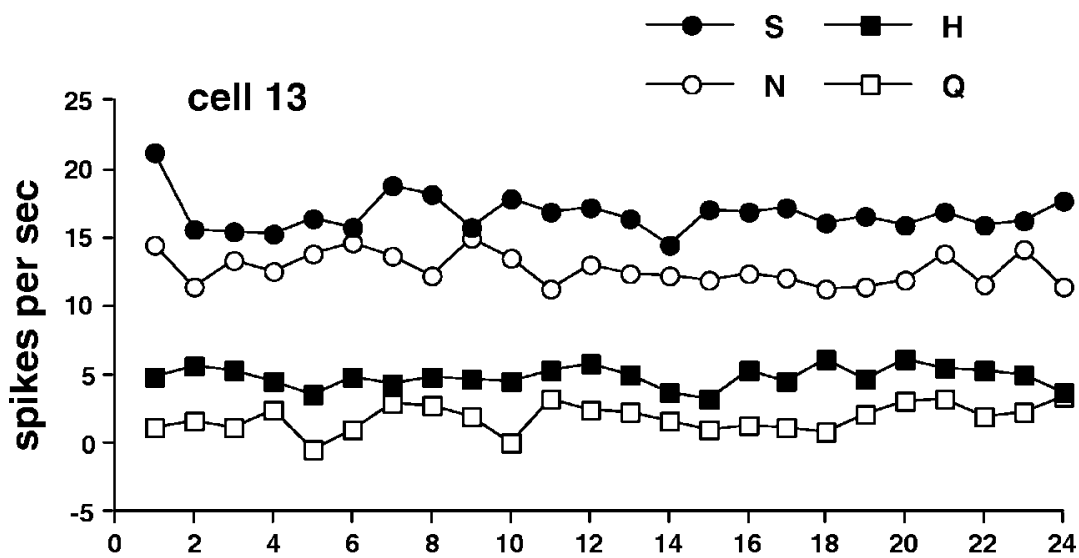

blocks of trials

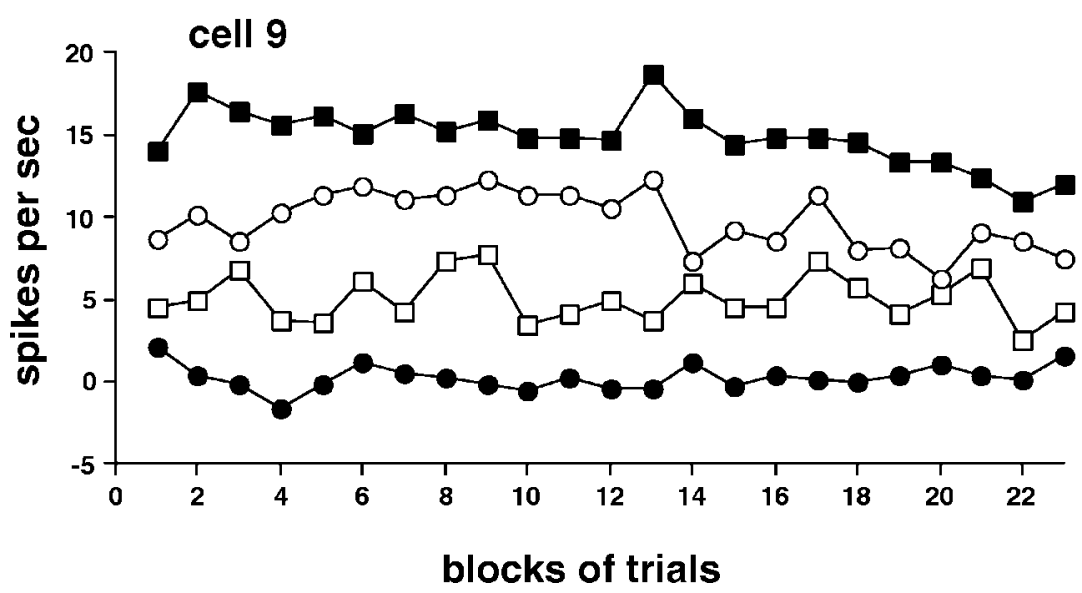

FIG. 2. Response magnitude with stimulus repetition in cells 13 and 9 where all 4 taste stimuli show consistently different response magnitudes. $\mathrm{S}$, sucrose; $\mathrm{N}, \mathrm{NaCl} ; \mathrm{H}, \mathrm{HCl}$; $\mathrm{Q}$, quinine. 

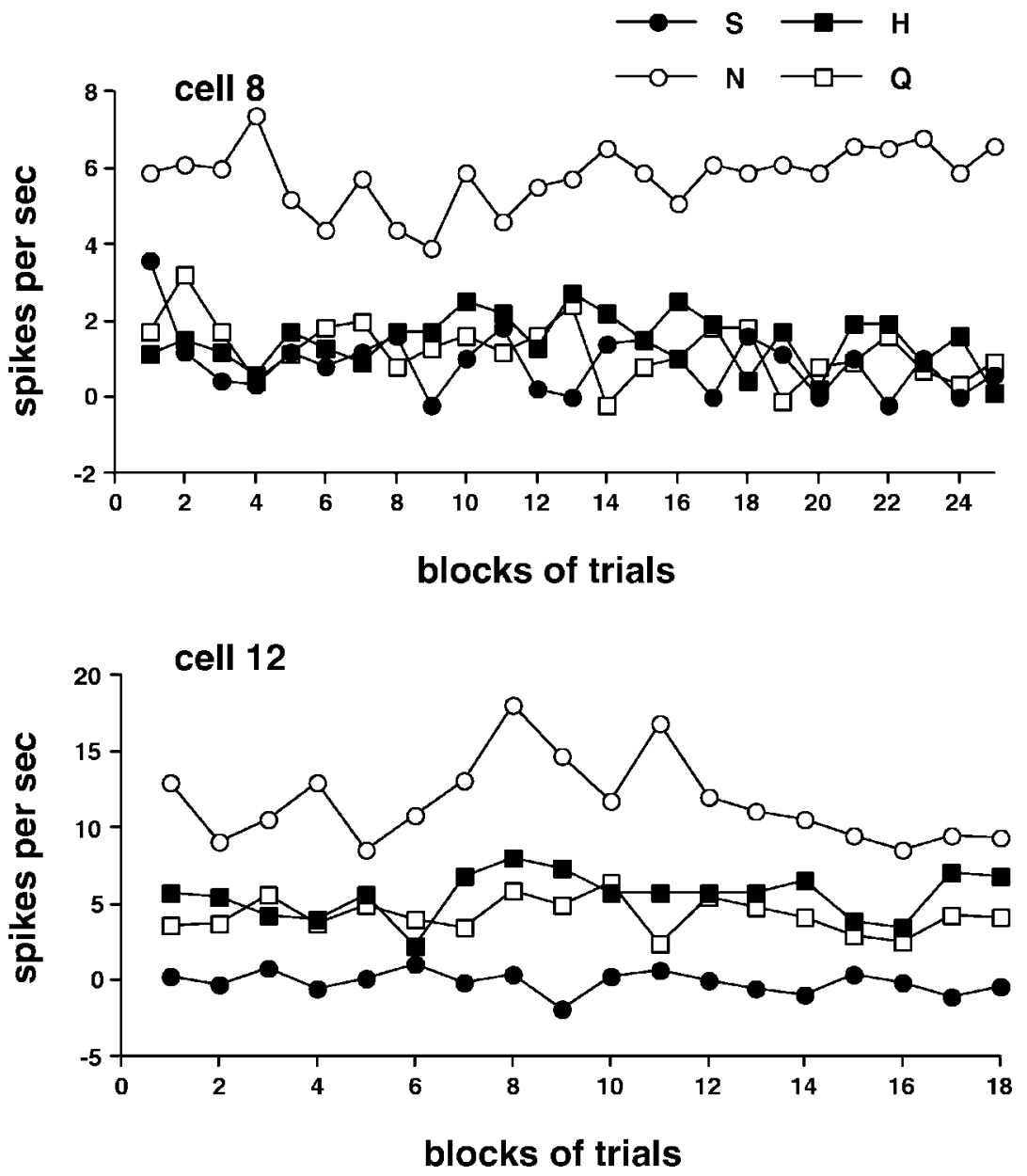

FIG. 3. Response magnitude with stimulus repetition in cells 8 and 12 where the response to the best stimulus is consistently different from the responses of the other tastants. shown in Figs. 2-4 are not intended to imply a strict typology among NTS cells. Rather they are presented to illustrate the various ways in which variability in response magnitude could impact the determination of the "best stimulus" of a cell as it is conceptualized in the taste literature.

Another direct result of the variability in response magnitude for many taste stimuli was a relatively large variation in the breadth of tuning, as measured by the uncertainty measure. Table 3 illustrates this point. For each cell, the uncertainty measure was calculated for each block of trials. The average as well as the minimum and maximum uncertainty values are shown. It can be seen that most cells can be characterized as either narrowly tuned (uncertainty value close to 0 ) or broadly tuned (uncertainty value close to 1.0 ) depending on the particular block of trials used in the calculation.

The average coefficient of variation ( $\mathrm{CV}$; SD/mean) for all taste responses was $0.31 \pm 0.03$. This measure was different for responses to different stimuli: for sucrose responses, $\mathrm{CV}=$ $0.39 \pm 0.13 ; \mathrm{NaCl}$ responses, $\mathrm{CV}=0.25 \pm 0.03 ; \mathrm{HCl}$ responses, $\mathrm{CV}=0.30 \pm 0.04$; and quinine responses, $\mathrm{CV}=$ $0.37 \pm 0.08$; however, these interstimulus differences were not statistically significant $[F(3,51)=1.285, P>0.05)$. Figure 5 shows the relationship of the mean response rate and the standard deviation for all significant responses. In general, responses of larger magnitude showed a smaller $\mathrm{CV}$ than responses of smaller magnitude. For example, the $\mathrm{CV}$ for responses that were 10 sps was $0.22 \pm 0.02$.
Temporal structure conveys information: individual examples

We first present the temporal coding analysis of three cells in detail and then describe the general characteristics of the population. Figure $6 A$ shows the post-stimulus histograms and $B$ shows a coding analysis of the discharges of one neuron during the 10-s period from stimulus presentation to rinse. The value of $H$ at $q=0, \sim 1$ bit, is the information extracted by a metric that only examines spike counts. As $q$ increases, the gradual increase in $H$ for $D^{\text {spike }}[q]$ and $D^{\text {interval }}[q]$ indicates that in addition to spike counts, the four tastants produce different temporal patterns in the responses and that these differences are sufficiently reliable to allow for greater discrimination of the tastants. The larger values of $H$ for $D^{\text {spike }}[q]$ as compared to $D^{\text {interval }}[q]$ indicates that the timing of the individual spikes, rather than the pattern of intervals, allows for this greater discrimination. Moreover, the eventual decline in $D^{\text {spike }}[q]$ and $D^{\text {interval }}[q]$ indicates that these temporal patterns are consistently related with the stimuli only up to a particular resolution. The highest value of the transmitted information is achieved by $D^{\text {spike }}[q]$ at $q_{\max }=4$. This means that at timescales shorter than the $1 / q_{\max }$ (i.e., $\leq 250 \mathrm{~ms}$ ), the timing of individual spikes no longer provides information concerning the identity of the stimulus. Finally, Fig. 6B, bottom, which summarizes parallel calculations on surrogate scrambled data sets, indicates that biases in the information estimates due to finite sample size is small. Figure $6 C$ shows the results of a similar analysis, confined to the initial $2 \mathrm{~s}$ after stimulus presentation. The coding 

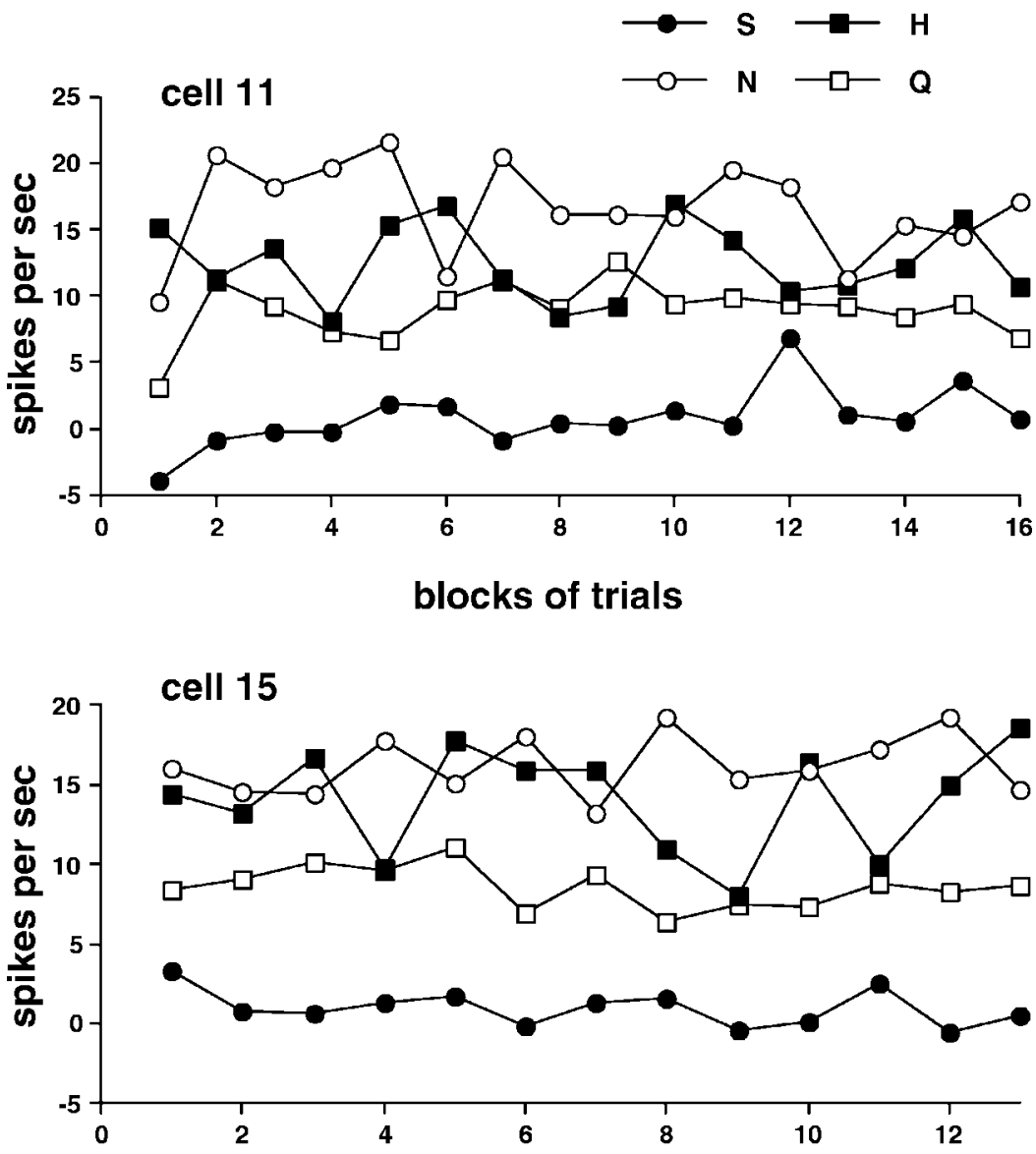

FIG. 4. Response magnitude with stimulus repetition in cells 11 and 15 where the best stimulus varies between $\mathrm{NaCl}$ and $\mathrm{HCl}$ across blocks of trials.

\section{blocks of trials}

characteristics identified in the first $2 \mathrm{~s}$ of response are similar to those seen in the entire 10-s response.

Figure $6 D$ shows the results of an "exchange resampling" of this data set. In this analysis, surrogate data sets are created that share the same overall firing rates and post-stimulus histograms of the original data by randomly exchanging spikes between

TABLE 3. Uncertainty of each cell

\begin{tabular}{cccc}
\hline \hline Cell & & Minimum & Maximum \\
\hline 1 & $0.59 \pm 0.02$ & 0.36 & 0.87 \\
2 & $0.78 \pm 0.02$ & 0.57 & 0.94 \\
3 & $0.72 \pm 0.02$ & 0.56 & 0.96 \\
4 & $0.77 \pm 0.03$ & 0.57 & 0.96 \\
5 & $0.72 \pm 0.02$ & 0.48 & 0.85 \\
6 & $0.83 \pm 0.02$ & 0.67 & 0.95 \\
7 & $0.46 \pm 0.04$ & 0.31 & 0.64 \\
8 & $0.7 \pm 0.03$ & 0.35 & 0.91 \\
9 & $0.75 \pm 0.02$ & 0.59 & 0.86 \\
10 & $0.76 \pm 0.01$ & 0.65 & 0.87 \\
11 & $0.79 \pm 0.03$ & 0.49 & 0.95 \\
12 & $0.71 \pm 0.02$ & 0.59 & 0.83 \\
13 & $0.82 \pm 0.01$ & 0.65 & 0.89 \\
14 & $0.81 \pm 0.02$ & 0.64 & 0.93 \\
15 & $0.81 \pm 0.02$ & 0.71 & 0.97 \\
16 & $0.81 \pm 0.04$ & 0.61 & 0.98 \\
17 & $0.93 \pm 0.01$ & 0.87 & 0.93 \\
19 & $0.73 \pm 0.02$ & 0.53 & 0.87 \\
22 & $0.77 \pm 0.01$ & 0.66 & $0.90 \pm 0.02$ \\
Average & $0.75 \pm 0.02$ & $0.57 \pm 0.03$ &
\end{tabular}

Values are means $\pm \mathrm{SE}$. trials of the same stimulus (Victor and Purpura 1996). There is no difference between the coding analysis of the original and the surrogate data sets as indicated by the overlapping curves. This indicates that the additional temporal information is fully contained in the time-dependent firing rates of the responses shown in Fig. 6A, and there is no additional contribution from the arrangement of spikes in individual trials.

Although the preceding characteristics were shared by many neurons, other behaviors, illustrated in Fig. 7, were also frequently seen. For this neuron, $H$ achieved its ceiling value of 2 bits for $q=0$, when the entire 10-s presentation period was considered (Fig. 7B). That is, each tastant led to a different level of activity, and the overall average firing rates for the four

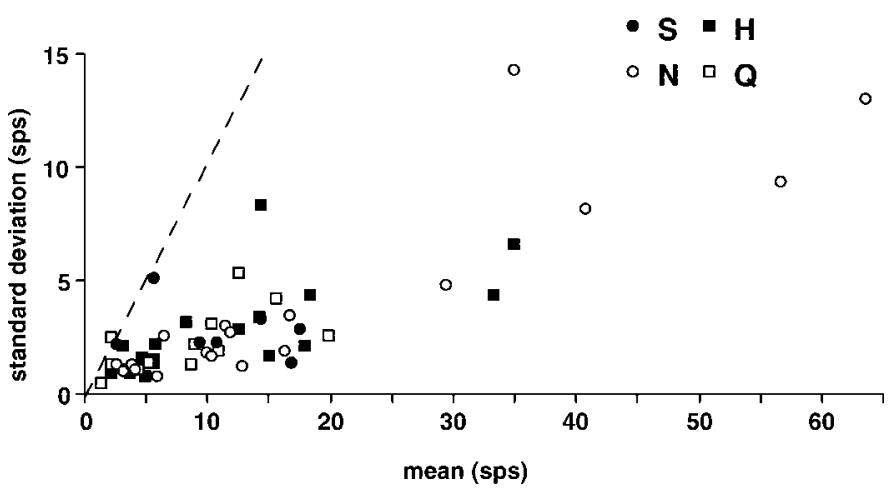

FIG. 5. Plot of mean response magnitude [spikes/s (sps)] vs. SD. - , condition where mean response magnitude equals SD. 
A
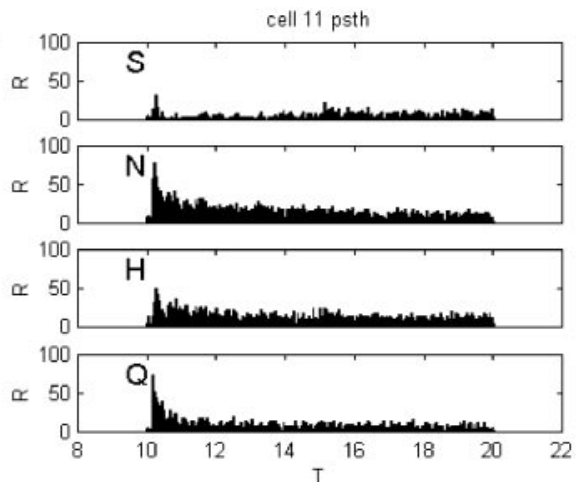

C

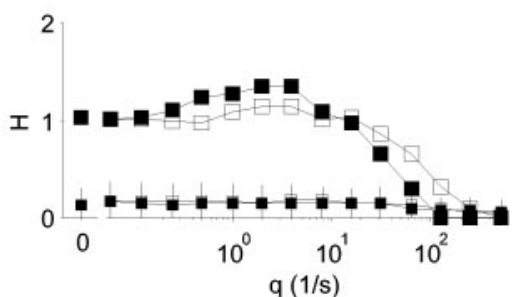

B

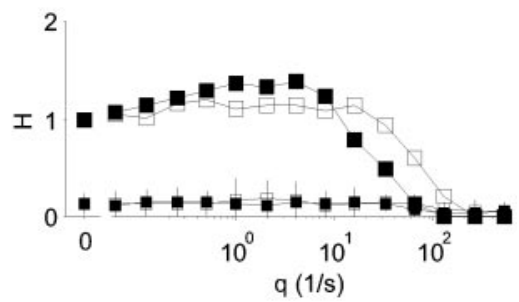

FIG. 6. Coding analysis of cell 11. A: poststimulus time histograms (PSTH) of responses to the 4 stimuli. Abbreviations are as in Fig. 2. $B$ : information-theoretic measure of response clustering, $H$, as a function of temporal precision $q$, for the 10-s response period. Solid squares, $D^{\text {spike }}[q]$; open squares, $D^{\text {interval }}[q]$. Large symbols, analysis of actual responses; small symbols, bias estimates derived from surrogate shuffled data sets. Error bars indicate 2 SD. $C$ : as in $B$, but analysis restricted to the initial $2 \mathrm{~s}$ of the response. $D$ : exchange resampling analysis of the 1 st $2 \mathrm{~s}$, comparing $H$ extracted from the actual responses via $D^{\text {spike }}[q]$ (solid squares) with $H$ extracted from surrogate data sets that matched the time-varying rate of the actual data (gray squares). In $B-D$, the abscissa is broken to indicate that the logarithmic transformation of $q$ does not extend down to 0 . tastants were sufficiently different so that they determined the stimulus nearly unambiguously. In contrast, when only the first $2 \mathrm{~s}$ of the presentation was analyzed (Fig. 7C), the information conveyed by spike counts alone dropped to $\sim 1.2$ bits. That is, during the first $2 \mathrm{~s}$ of response, there was sufficient overlap in mean firing rates for the four tastants, and/or the trial-to-trial variability was sufficiently large, so that firing rate alone could not disambiguate the stimuli. When the same response segments are analyzed with metrics that are sensitive to the firing of individual spikes, the information that can be extracted rises to $\sim 1.8$ for $D^{\text {spike }}[q]$ at $q_{\max }=4$. As in the cell of Fig. 6 , this indicates that spike timing, at a resolution of $\sim 1 / q_{\max }(250 \mathrm{~ms})$ are reliably linked to the stimulus and provide information not present in the average firing rate. Examination of the poststimulus time histograms (Fig. 7A) suggests that some of this benefit may be due to the slightly earlier response latency, and/or shorter response transients, associated with tastants $\mathrm{Q}$ and $\mathrm{H}$. (Discrimination of tastants $\mathrm{N},\{\mathrm{Q}, \mathrm{H}\}$, and $\mathrm{S}$, but complete confusion of $\mathrm{Q}$ and $\mathrm{H}$, would correspond to an information of 1.5 bits). In contrast to the cell of Fig. 6, the interval structure does not contribute to coding, as seen by the lack of increase in $D^{\text {interval }}[q]$ as $q$ rises. Another difference is revealed by the exchange resampling analysis of Fig. $7 D$. In the neighborhood of $q_{\max }=4$, the information conveyed by the actual responses is significantly higher than the information conveyed by responses that are matched for time-varying firing rate but otherwise random. That is, the temporal structure of the actual responses is not adequately described by a time-varying Poisson process, and the additional structure of the actual responses (i.e., the arrangement of individual spikes in individual trials) is informative.
A
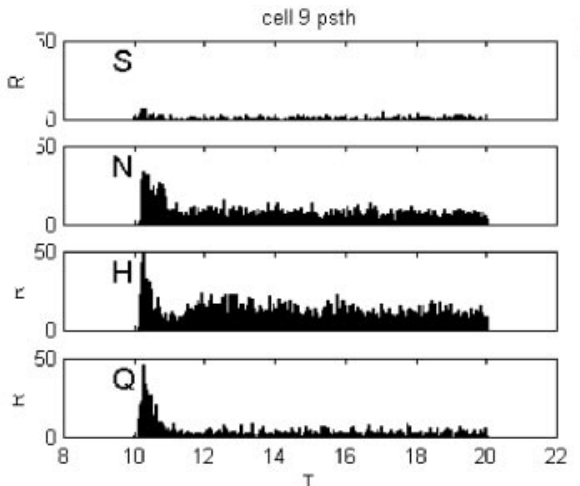

C

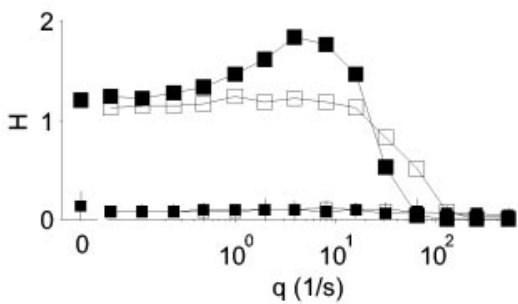

B

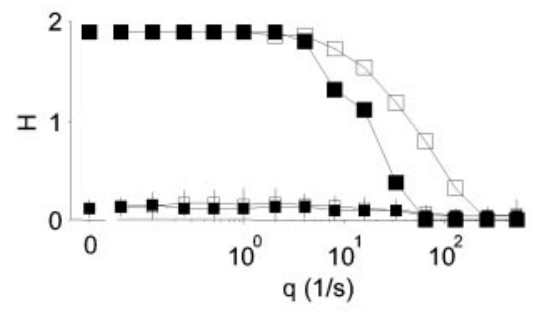

FIG. 7. Coding analysis of cell 9. Details as in Fig. 6. 
A
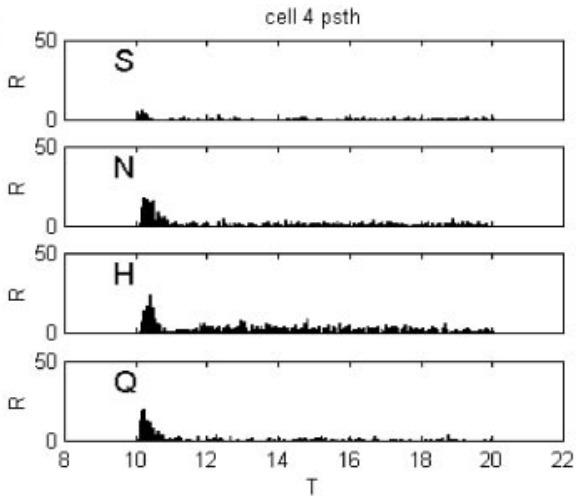

C

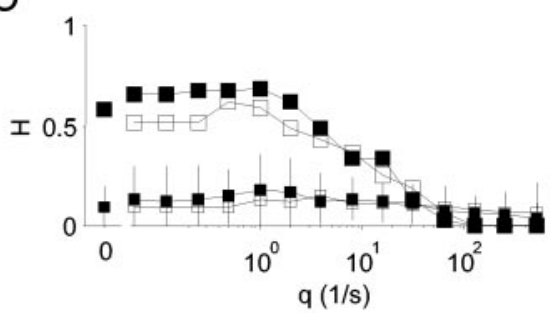

B

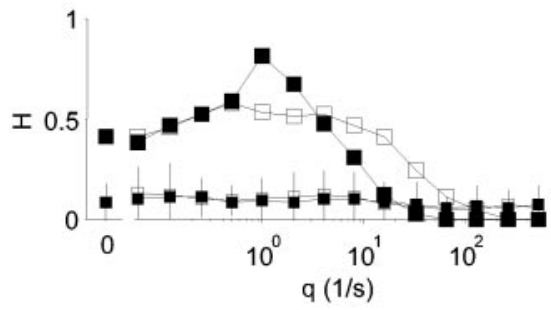

FIG. 8. Coding analysis of cell 4. Details as in Fig. 6.
D

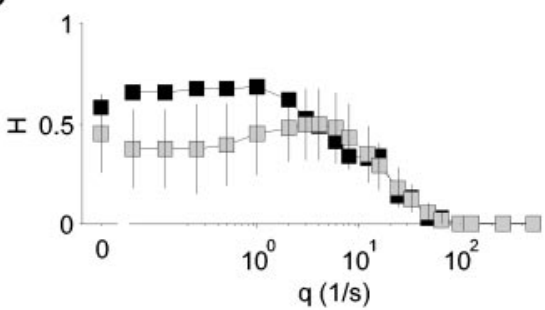

A third variation is shown by the neuron illustrated in Fig. 8 . This neuron shows evidence of temporal coding, as seen by the increasing values of $H$ as $q$ rises from 0 in Fig. 8, $B$ (the entire response) and $C$ (the $1 \mathrm{st} 2 \mathrm{~s}$ of the response). As in the example of Fig. 7, this increase is seen for the metric based on spike times but not for the metric based on interspike intervals. In this neuron, temporal coding made a significant contribution when the entire response period was examined (10-20 s, Fig. $8 B$ ) but not when the first $2 \mathrm{~s}$ of the response was examined $(10-12 \mathrm{~s}$, Fig. 8C). This is in contrast to the behavior of the cell of Fig. 6 (which showed a modest contribution of temporal coding in both analysis intervals) and the cell of Fig. 7 (which only showed a contribution of temporal coding in the $1 \mathrm{st} 2 \mathrm{~s}$ ). As in the cell of Fig. 7, the exchange analysis indicated that the temporal structure of the actual responses is not adequately simulated by a time-varying Poisson process.

Although the differences in amounts of information discussed in the preceding text might be considered small $(0.2-$ 0.5 bits), this should be viewed in the context of the fact that spike counts already provide $\sim 1$ bit of information in most cases, and the use of only four stimuli limits the maximum possible amount of information to 2 bits. A rigorous calculation of the bias and uncertainty of the information estimates is problematic even via non-parametric statistics such as the jackknife or the bootstrap (Efron and Tibshirani 1998) because a technical requirement, continuity of the statistic in the data, is violated. Nevertheless, the shuffle resamples and their error bars (small symbols in $B-D$ of Figs. 6-8) provide an approximate bias and uncertainty of our estimates. By this criterion, the phenomena described in the preceding text were all $>2$ SD.

\section{Temporal structures that convey information: population summary}

Figures 9-11 summarize the coding analysis across the population of recorded neurons. Figure 9 compares information transmitted by codes that use spike times and interspike intervals to the information transmitted by spike counts alone.
For both the entire 10-s response and the 2 -s response, both temporal codes convey more information than a spike count code. This difference is greater for the spike time codes than for spike interval codes $(10-20 \mathrm{~s}, A$ vs. $B, P<0.003$ via 2-tailed paired $t$-test; $10-12 \mathrm{~s}, C$ vs. $D, P<0.0002$ via 2 -tailed paired $t$-test). There was also a tendency for the contribution of spike timing to be larger in the first $2 \mathrm{~s}$ of the response than for the entire response period ( $A$ vs. $C, P=0.15$ via 2-tailed paired $t$-test).

Figure 10 compares the information conveyed in the entire

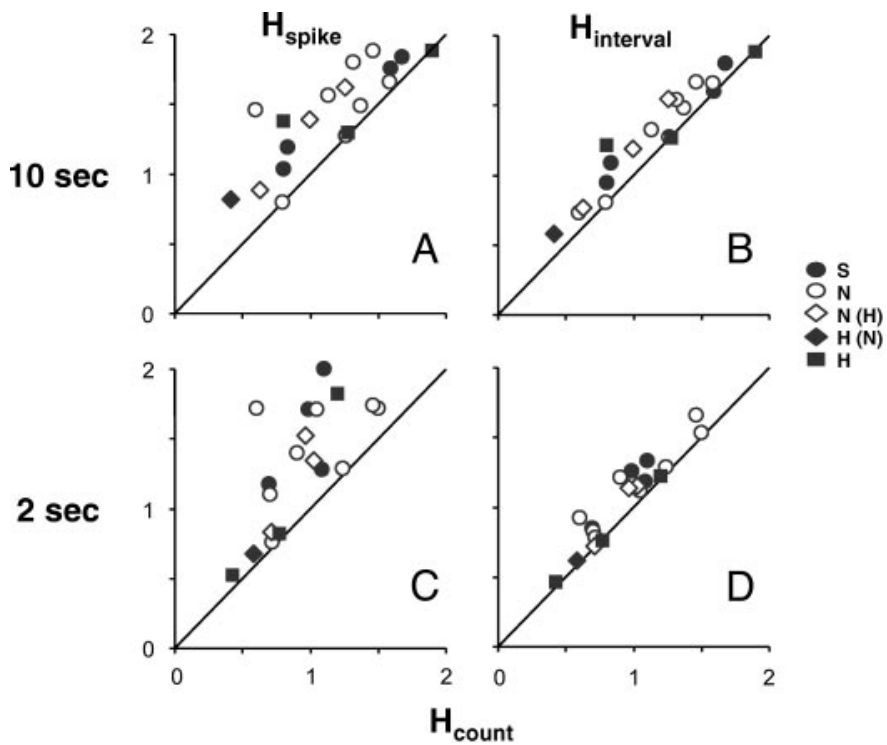

FIG. 9. Contribution of spike counts, spike times, and spike intervals to coding. $A$ and $B$ : the entire 10 -s response; $C$ and $D$ : the 1 st 2 s. $A$ and $C$ compare information extracted by spike counts alone (abscissa) with information extracted by $D^{\text {spike }}\left[q_{\max }\right] . B$ and $D$ compare information extracted by spike counts alone (abscissa) with information extracted by $D^{\text {interval }}\left[q_{\max }\right]$. Each point corresponds to an individual cell. The key indicates the best stimulus on the basis of the average measure $[\mathrm{S}, \mathrm{N}, \mathrm{H}]$, or, for cells whose preference by the average measure and the first measure differed, based on average (and first) measure $[\mathrm{N}(\mathrm{H}), \mathrm{H}(\mathrm{N})]$. 


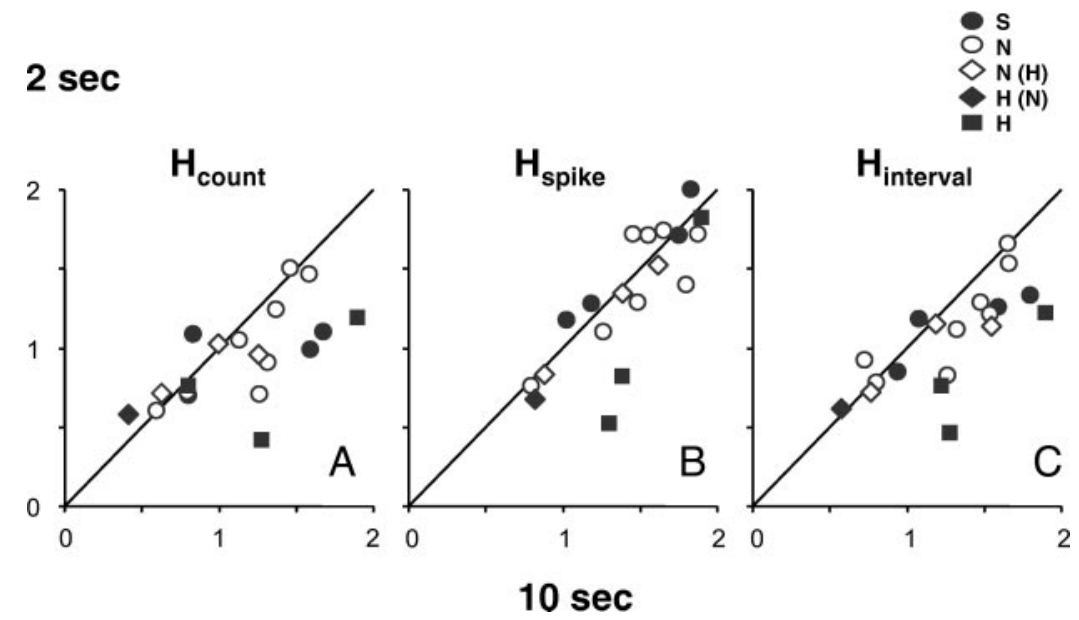

FIG. 10. Comparison of importance of temporal coding for the 10-s response (abscissa) and for the $1 \mathrm{st} 2 \mathrm{~s}$ of response (ordinate). A: spike counts. $B: D^{\text {spike }}\left[q_{\max }\right] . C: D^{\text {interval }}\left[q_{\max }\right]$. Data are replotted from Fig. 9 with the same conventions for symbols.

10 -s response with information conveyed in the initial $2 \mathrm{~s}$. Not surprisingly, the entire response does convey more information than the first $2 \mathrm{~s}$ of response. However, this difference is the smallest for the spike time codes $(B)$. Together, Figs. 9 and 10 show that timing contributes substantially to coding, especially during the first $2 \mathrm{~s}$ of response. [In Fig. 10, the points that lie slightly above the diagonal suggest that more information is available in the $1 \mathrm{st} 2 \mathrm{~s}$ than in the full $10 \mathrm{~s}$ of the response. This would appear to violate the data processing inequality (Cover and Thomas 1991), which implies that information cannot decrease when a response is truncated. The reason for this apparent violation is that ordinate and abscissa represent the amount of information that can be extracted from via a particular set of decoding strategies, not the total information actually present. If the decoding strategies are suboptimal, then the amount of information that they extract can increase when portions of the response are deleted. A related apparent violation of the data processing inequality is the decline in the information curves for sufficiently high $q$.]

The exchange resampling analysis, summarized in Fig. 11, shows that for many of the neurons studied, the information conveyed by spike timing was more than that conveyed by an equivalent modulated Poisson process (i.e., coding via a timevarying rate). This difference was significant $(P<0.05$ via 2 -tailed $t$-test) in 2 of the 19 cells when analyzed over the entire response $(A)$ and in 10 of the 19 cells when analyzed over the first $2 \mathrm{~s}$ of the response $(B)$.

In general, the coding behavior of a cell appeared to be

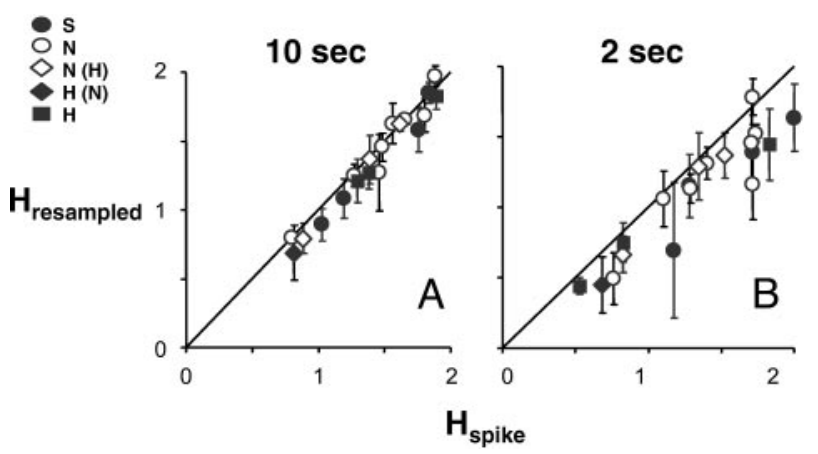

FIG. 11. Summary of exchange resampling analysis for $D^{\text {spike }}\left[q_{\max }\right]$ for entire 10-s response $(A)$ and 1 st $2 \mathrm{~s}(B)$. Abscissa: $H$ as determined from actual data. Ordinate: $\mathrm{H}$ as determined from surrogate datasets matched for timevarying rate. Error bars: 2 SD. Conventions for symbols as in Fig. 9. independent of its preferred stimulus. Although only a relatively small population of cells was analyzed, the population contained cells with $\mathrm{S}, \mathrm{N}$, and $\mathrm{H}$ preferences distributed throughout the scattergrams of Figs. 9-11. Values of $q_{\max }$, which indexes the temporal precision of the code, varied widely throughout the population, from 0 to 16 (considering the entire 10-s response) and 1 to 8 (considering the first $2 \mathrm{~s}$ ) and also did not correlate with the preferred stimulus.

Table 4 shows the $\mathrm{CV}$ for all significant responses for each cell as well as a measure of the relative increase in the information conveyed by spike timing $\left(D^{\text {spike }}\right)$ and by the intervals between spikes $\left(D^{\text {interval }}\right)$. The latter measure was calculated as the arithmetic difference between the information conveyed by the spike count and the maximum information conveyed by either spike timing or ISIs divided by the information conveyed by the spike count. So for either $D^{\text {spike }}$ or $D^{\text {interval }}$, the percent increase in information (\%incr) was calculated as

$$
\text { \%incr }=\frac{H_{\max }-H_{\text {count }}}{H_{\text {count }}} \times 100
$$

where $H_{\text {count }}$ is the information conveyed by spike count and $H_{\max }$ is the maximum amount of information conveyed by either $D^{\text {spike }}$ or $D^{\text {interval }}$. It is an index of the proportional increase in the amount of information conveyed when either spike timing or ISIs are used to discriminate among tastants compared with the information conveyed by spike count alone. There is a strong correlation between this measure and the CV for $D^{\text {spike }}(r=0.85, \mathrm{df}=17, P<0.01)$ and $D^{\text {interval }}(r=0.58$, $\mathrm{df}=17, P<0.01)$ when the entire $10-20 \mathrm{~s}$ of response is considered. This suggests that the more variable the response magnitude within a cell, the more informative are the timing of spikes and the sequence of ISIs in terms of discriminating among the four tastants.

\section{Discussion}

Electrophysiological responses to representatives of the four basic taste qualities were recorded from 19 cells in the NTS of anesthetized rats. Presentation of each stimulus was repeated a minimum of 8 and a maximum of 29 times for each cell. Analyses of reliability of responses across several repetitions of stimulus presentation showed that response magnitude to a given tastant varied widely within some NTS cells. As a result, the breadth of tuning across stimuli, as it is traditionally cal- 
TABLE 4. Response variability and analysis of temporal coding for individual NTS cells

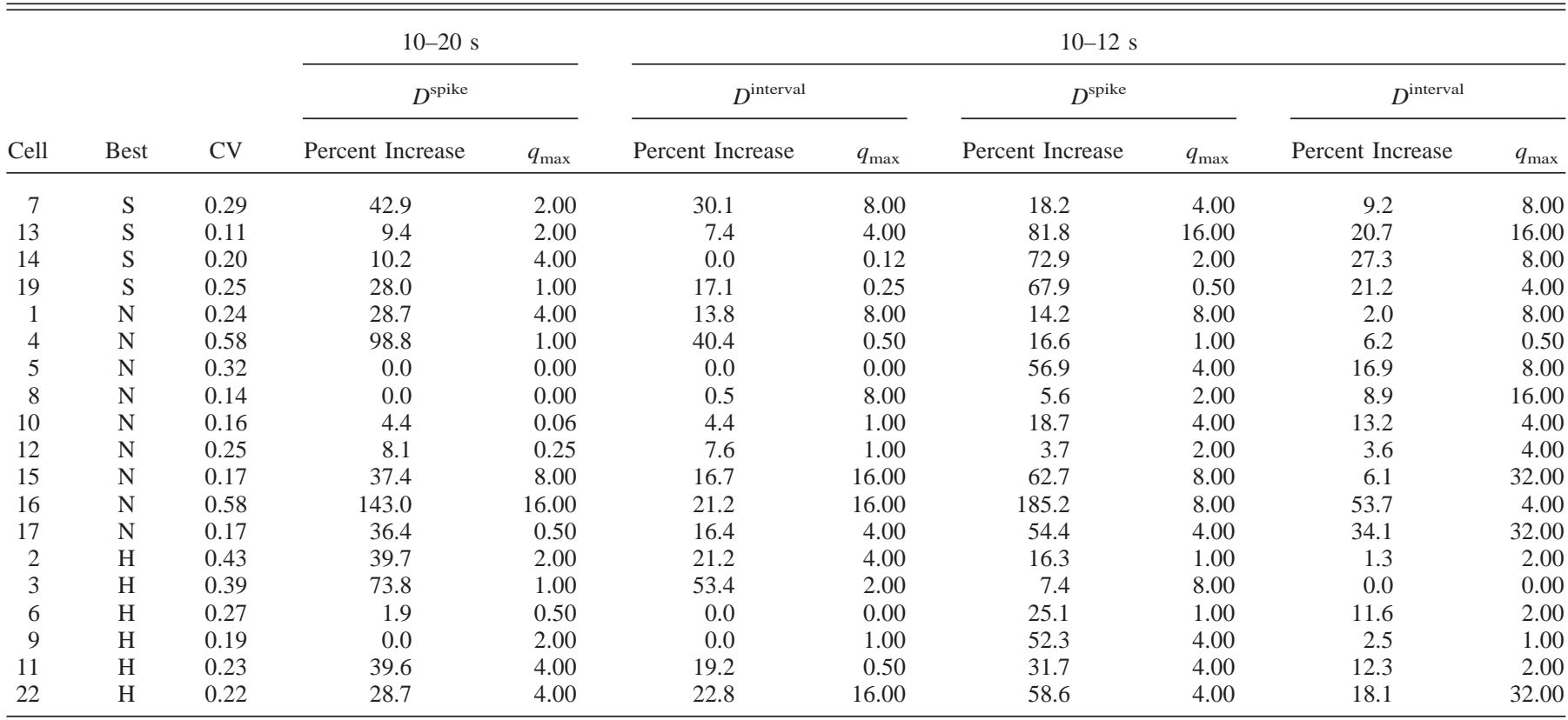

$\mathrm{CV}$, average coefficient of variation for each cell; Best, best stimulus determined by the first four stimulus presentations; $\mathrm{S}$, sucrose, $\mathrm{N}, \mathrm{NaCl}, \mathrm{H}, \mathrm{HCl}$. Percentage increase was calculated as $\left[\left(H_{\max }-H_{\text {count }}\right) / H_{\text {count }}\right] * 100$ for each metric and at each interval of analysis. $q_{\max }$ is the value of $q$ when $H$ was at its maximum value.

culated, for a given cell could be large or small depending on the particular responses used for calculation or the details of how data from repeated responses are combined. Given the observed variability in response magnitude, determination of the true breadth of tuning of a taste cell may require the inclusion of many stimulus replications. On the other hand, most but not all NTS cells had the same best stimulus regardless of the method of determination. For the remaining cells, small differences among response magnitudes may indicate that large numbers of trials are necessary to identify the best stimulus. Alternatively, some cells may be inherently broadly tuned, with no clear best stimulus. These cells might correspond to the generalist fibers that have been identified in peripheral taste nerves (Frank et al. 1983, 1988). Analyses of the contribution of the temporal parameters of taste responses showed that spike timing significantly contributes to the discrimination of taste qualities in 10 of $19(53 \%)$ cells. This contribution was especially notable during the initial $2 \mathrm{~s}$ of the response. In these cells, observation of the temporal pattern of the response makes it possible to distinguish the identity of the stimulus more accurately than merely counting the number of spikes.

Our analysis not only demonstrates the magnitude of the temporal contribution to coding but at least partially characterizes what aspects of the temporal pattern are relevant. The temporal contribution to coding rests on the times of individual spikes, rather than the pattern of intervals. This is seen by a comparison of the information curves for $D^{\text {spike }}[q]$ and $D^{\text {interval }}[q]$ in $B$ and $C$ of Figs. $6-8$ and by a comparison of Fig. 9's columns, which compare the peak values of these curves. Temporal pattern matters more in the first $2 \mathrm{~s}$ of the response, than across the entire $10 \mathrm{~s}$ of the response. This is seen by a comparison between $B$ (all $10 \mathrm{~s}$ ) with $C$ (1st $2 \mathrm{~s}$ ) in Figs. 6-8, and a comparison of the rows of Fig. 9.
The time scales identified here (several hundred $\mathrm{ms}$ ) are greatly in excess of times relevant for neural coincidence detection, typically on the order of a few msec (Cline 1997; König et al. 1996; Softky 1994). Thus it is unlikely that the temporal structures that we have identified are decoded simply by coincidence detectors per se. Rather cellular and circuit mechanisms with much longer time constants must play a role.

The position of the peak of the information curve for $D^{\text {spike }}[q]$, denoted $q_{\max }$, indicates the time scale $\left(1 / q_{\max }\right)$ at which spike timing is informative. That is, if a spike is shifted by an amount that is comparable to $1 / q_{\max }$, the "message" of the spike train changes just as much as if the spike were added or deleted. In this population of cells, analysis of the first $2 \mathrm{~s}$ of the response led to values of $q_{\max }$ with a geometric mean of 3.2 and a range of 1-16. The geometric mean corresponds to a temporal precision of $\sim 300 \mathrm{~ms}$, but the upper end of the range indicates that in some cells, shifting the time of a spike by ca. $60 \mathrm{~ms}(=1 / 16)$ changes its "meaning" significantly. When the entire response was analyzed, the geometric mean value of $q_{\max }$ dropped to 1.1, indicating that spike timing during the first two $\mathrm{s}$ of response was substantially more precise than over the entire response.

The final aspect of the analysis ( $D$ of Figs. 6-8; Fig. 11) determined whether the information carried by spike times could be accounted for by a Poisson spike train whose rate varied according to the observed average PSTH. To do this, we constructed surrogate data sets of modulated Poisson spike trains that shared the same PSTHs as the actual data and also had the same number of spikes per trial (exchange resampling, Victor and Purpura 1996). In 10 of 19 cells, the actual data sets carried more information than these surrogate data sets. This implies that temporal coding is not merely that of a timevarying firing rate. In other words, spikes are not just an 
estimator of firing rate, and the placement of spikes in individual trials is informative.

It is interesting to compare this analysis with a corresponding analysis of coding of visual attributes by neurons in macaque visual cortex (Victor and Purpura 1996). As in the present study, those analyses indicated a substantial contribution of temporal coding, carried primarily by spike times, rather than spike intervals. However, temporal coding of visual attributes was at a much finer time scale, with typical values of $q_{\max }$ ranging from 10 to 100 (larger values for coding contrast, smaller values for coding various aspects of visual form). A further analysis of coding of contrast (Reich et al. 2001) demonstrated that, as in the present study, the precision of the temporal code during the response transient was substantially higher than during the remainder of the response. Finally, the extent to which temporal coding is not merely a manifestation of a time-varying firing rate was more prominent in the present study than in the study of macaque visual cortex, where the contribution was only minimally significant across several hundred data sets (Victor and Purpura 1996).

\section{Response variability and taste coding}

The observation that response magnitude can vary widely within some NTS cells compliments similar data on response variability of chorda tympani nerve responses reported by Ogawa et al. (1973). In that study, variability of taste-response magnitude in eight chorda tympani fibers was studied over six stimulus repetitions. Like the present data in the NTS, taste responses in some chorda tympani fibers showed large variation in response magnitude while others remained relatively constant. The $\mathrm{CV}$ in that study ranged between 0.1 and 0.25 which is somewhat smaller than that reported here, suggesting that NTS cells show more variable response magnitudes than their inputs. Considering the well-known finding that NTS cells are more broadly tuned than chorda tympani fibers (Doetsch and Erickson 1970), the observation that response rates are more variable than those in chorda tympani fibers suggests that coding mechanisms besides, or perhaps in addition to, rate coding may come into play. These mechanisms include across neuron pattern coding as well as temporal coding. The idea that variability in response magnitude is compensated by the availability of other coding mechanisms is supported by the observation that variability of response magnitude (as measured by the CV) is significantly correlated with the proportion of the total information conveyed by spike timing and ISIs. That is, it is possible that the timing of spikes is a more reliable and informative aspect of a response in those cells where the spike count is very variable.

In the labeled line theory of taste coding, the best stimulus of a cell, i.e., the stimulus that evokes the most vigorous response, defines the role of that cell in the coding process. For example, $\mathrm{NaCl}$-best cells are thought to encode saltiness, sucrose-best cells are thought to encode sweetness, etc. Neurons with a particular best stimulus are thought to respond in predictable proportion to other, non-best tastants. This observation has fueled arguments that best stimulus classes represent functional neuron types. If labeled line coding was used in the brain stem, then it would be reasonable to expect that the best stimulus of a cell would be readily apparent from a single trial. Results from the present study, however, showed that the best stimulus, as measured in several different ways, was unambiguous for some but not all NTS cells. Though the best stimulus was identical on each set of trials for the majority of NTS cells (12 of $19 ; 63 \%)$, the response magnitudes of seven cells (37\%) had sufficient variability so that the apparent best stimulus vacillated. Even in some cells that showed an unambiguous best stimulus, the responses to the non-best stimuli were unpredictable and overlapping. Overall, these data suggest that there is some proportion of cells that might be eligible to participate in a labeled line code based solely on rate coding by virtue of their reliable response rates. However, it is difficult to reconcile labeled line coding with the variability in response magnitude that we observed in the remaining cells.

\section{Periodicity of response rate for sucrose responses}

From some of the earliest reports of time course analyses of sucrose responses in the peripheral nerves, the responses to sucrose have been described as periodic bursts of spikes (Fishman 1957; Mistretta 1972; Nagai and Ueda 1981; Ogawa et al. 1973, 1974). These bursts have been observed to occur every 200-300 ms over the course of the response. In the NTS, however, in a detailed analysis of the temporal patterns of response, Travers and Norgren (1989) failed to find convincing evidence for such periodicity. In agreement with their finding, examination of ISI and PSTHs of sucrose responses in the present study found no evidence for rhythmicity in firing rate. Additionally, we did not find that characteristics of temporal coding in neurons whose best stimulus was sucrose differed from those of the rest of the recorded neurons.

\section{Time course of response and discrimination of taste stimuli}

Most, if not all, descriptions of the time course of taste responses in the nervous system indicate that there is an initial "phasic" portion of the response, followed by a decrease in firing rate to a stable, "tonic" level that remains above spontaneous rate. This pattern is seen in anesthetized animals where tastants are passively bathed over the tongue and in awake animals that are licking (Nishijo and Norgren 1991). The duration of the initial phasic portion of the response has been variously described as lasting from $0.2 \mathrm{~s}$ (e.g., Doetsch and Erickson 1970) to 2 s (e.g., Di Lorenzo and Schwartzbaum 1982) from the initiation of the response. Because it is known that rats can make behavioral decisions about taste stimuli within the first $1 \mathrm{~s}$ of a response (Halpern 1985; Halpern and Tapper 1971; Scott 1974), the initial phasic portion of the response is thought to have special significance in taste discrimination. Accordingly, several investigators have analyzed the phasic and tonic portions of taste responses separately (Di Lorenzo and Schwartzbaum 1982; Nagai and Ueda 1981), whereas others have examined the ratio of phasic to tonic portions of the response (Scott and Mark, 1989; Travers and Norgren 1989; Verhagen et al. 2003).

Results of the present study suggest that information about taste stimuli is most likely encoded by a different combination of mechanisms in the initial portion of the taste response compared with mechanisms employed later in the response interval. More specifically, results showed that the information conveyed by spike timing was most prominent in the first $2 \mathrm{~s}$ of response and less so when the entire $10 \mathrm{~s}$ response interval 
was analyzed. When the full $10 \mathrm{~s}$ of response was examined, only two cells showed a significant increase in information transfer when temporal coding was considered along with rate coding. However, when the same analysis was conducted on the first $2 \mathrm{~s}$ of response, 10 of 19 cells showed an advantage to including spike timing as a coding mechanism in the transmission of information about taste stimuli. These results suggest that information conveyed by the temporal parameters of the response, in particular by spike timing, is most influential in this initial response interval; however, when longer response intervals are considered, temporal coding mechanisms may become less informative than rate coding. This is not meant to imply that there are two distinct phases of a response each of which utilizes a distinct coding mechanism. Rather these data point to a qualitative change in the response characteristics over time.

Though the present results imply that temporal coding in the NTS may be used to identify taste stimuli, several lines of evidence support the hypothesis that temporal coding in the initial portion of the response conveys information about the hedonic value (an index of acceptance or rejection) of a taste stimulus. For example, principal component analyses of the time course of response in the parabrachial pons of the rabbit have shown that the information conveyed by temporal pattern of response alone in the first $2 \mathrm{~s}$ of response was sufficient only to distinguish between tastants of different hedonic value. More recently, based on analyses of the time course of response in relation to stimulus toxicity, Verhagen et al. (2003) have suggested that the ratio of the phasic to tonic portions of taste responses in the brain stem and thalamus relate to the hedonic characteristics of a taste stimulus. Moreover, Di Lorenzo and Hecht (1993) demonstrated that the temporal pattern of $1 \mathrm{~s}$ trains of lick-contingent electrical stimulation of the NTS in awake rats could predict acceptance or rejection depending on the stimulus used as a template for the stimulation.

\section{Different roles for individual cells in taste coding}

One of the clear results of the present study was the finding that some cells utilize temporal coding to a greater degree than others. In fact, there were some cells that showed no evidence of temporal coding at all. This result is in agreement with several other investigations showing that unique temporal organization of taste responses is a function of the cell rather than the stimulus. In the CT (Mistretta 1972; Ogawa et al. 1973, 1974) and superior laryngeal (Bradley et al. 1983) nerves, it has been shown that stimulus-related variations in the time course of response are idiosyncratic across fibers. Further, in both the CT nerve (Nagai and Ueda, 1981; Ogawa et al 1974) and NTS (Nuding et al. 1991), distinctive ISI distributions associated with various taste stimuli vary according to the cell and are not consistent across cells. Additionally, in the gustatory cortex, not every cell distinguishes among tastants with temporal modulation of firing rate (Katz et al. 2001).

The heterogeneity of the pool of taste responsive cells in the NTS in terms of the use of coding mechanisms may correlate with input-output relationships as well as the type of information that is encoded. As discussed in the preceding text, there is a case to be made that temporal coding, especially in the phasic portion of the response, may be used to convey hedonic value. This type of information may be relayed to the reticular formation (DiNardo and Travers 1997; Travers and Hu 2000) where it is used to orchestrate appropriate orofacial reflexes. Conversely, information about taste quality and intensity may be preferentially relayed rostrally along the main gustatory pathway. Because there is no overlap between those cells projecting rostrally to the $\mathrm{PbN}$ or ventrally to the reticular formation (Halsell et al. 1996), it is tempting to suggest that the target cells in the reticular formation receiving NTS output are especially well tuned to respond to unique temporal patterns of input. This is an empirical question that awaits experimental confirmation.

\section{Conclusions}

Results of the present experiment have demonstrated that taste responses in the brain stem can, at times, vary widely in magnitude with repetition. This variability provides a cautionary note for investigators who rely on single trials of each taste stimulus. Specifically, present data suggest that the true breadth of tuning, and perhaps also the best stimulus, of a taste cell may only be revealed by examination of several repetitive stimulus presentations.

Further analyses of taste responses showed that some brain stem cells can communicate about the four basic taste stimuli with different temporal patterns of response. In particular, those cells with responses that were most variable in magnitude over several repetitions were those that showed the largest contribution of temporal coding. Overall, temporal coding plays a larger role in the initial response interval, i.e. the first $2 \mathrm{~s}$, than in later portions of the response. This goes beyond the intuition that the precision of spike timing is most sharp during the initial phasic portion of the response in that it implies that the differences between the timing of the phasic responses elicited by distinct stimuli are reliable cues to the identity of the stimulus.

To determine the extent to which the temporal parameters of the response can uniquely specify a particular taste stimulus, it will be necessary to record and analyze the responses to a larger array of taste stimuli than were used here and at a variety of concentrations. Because numerous replications will be necessary to obtain reliable results, these experiments will be technically challenging.

The authors thank S. Vadlamudi for substantial technical contribution to this manuscript.

\section{IS C L OS URES}

This work was supported by National Science Foundation Grant BNS0077965 to P. M. Di Lorenzo and National Eye Institute Grant EY-9314 to J. D. Victor.

\section{REFERENCES}

Bradley RM, Stedman HM, and Mistretta CM. Superior laryngeal nerve response patterns to chemical stimulation of sheep epiglottis. Brain Res 276: 81-93, 1983

Cariani PA. As if time really mattered: temporal strategies for neural coding of sensory information. Commun Cognit Artifi Intell 12: 161-229, 1995.

Cline H. Coincidence detection in the nervous system. Trends Neurosci 19: 566-567, 1997. 
Cover TM and Thomas JA. Elements of information theory. In: Wiley Series in Telecommunications, edited by Schilling DL. New York: Wiley, 1991, p. 542.

Covey E. Temporal Coding in Gustation (Doctoral dissertation). Durham, NC: Duke University, 1980.

DeAngelis GC, Ohzawa I, and Freeman RD. Receptive-field dynamics in the central visual pathways. Trends Neurosci 18: 451-458, 1995.

Di Lorenzo PM and Hecht GS. Perceptual consequences of electrical stimulation in the gustatory system. Behav Neurosci 107: 130-138, 1993.

Di Lorenzo PM and Schwartzbaum JS. Coding of gustatory information in the pontine parabrachial nuclei of the rabbit: temporal patterns of neural response. Brain Res 251: 245-257, 1982.

DiNardo LA and Travers JB. Distribution of fos-like immunoreactivity in the medullary reticular formation of the rat after gustatory elicited ingestion and rejection behaviors. J Neurosci 17: 3826-3839, 1997.

Doetsch GS and Erickson RP. Synaptic processing of taste-quality information in the nucleus tractus solitarius of the rat. J Neurophysiol 33: 490-507, 1970.

Efron B and Tibshirani RJ. An Introduction to the Bootstrap. Monographs on Statistics and Applied Probability, edited by D. R. Cox, et al. Boca Raton, FL: Chapman \& Hall/CRC Press, vol 57, 436 pgs.

Eggermont JJ. Is there a neural code? Neurosci Biobehav Rev 22: 355-370, 1998.

Fishman IY. Single fiber gustatory impulses in rat and hamster. J Cell Comp Physiol 49: 319-334, 1957.

Frank M. An analysis of hamster afferent taste nerve response functions. J Gen Physiol 61: 588-618, 1973.

Frank ME. Neuron types, receptors, behavior, and taste quality. Physiol Behav 69: 53-62, 2000.

Frank ME, Beiber SL, and Smith DV. The organization of taste sensibilities in hamster chorda tympani nerve fibers. J Gen Physiol 91: 861-896, 1988

Frank ME, Contreras RJ, and Hettinger TP. Nerve fibers sensitive to ionic stimuli in chorda tympani of the rat. J Neurophysiol 50: 941-960, 1983.

Funakoshi M and Ninomiva Y. Neural code for taste quality in the thalamus of the dog. In: Food Intake and the Chemical Senses, edited by Katsuki Y, Sato M, Takagi SF, and Oomura Y. Tokyo, Japan: University Park Press, 1977, 223-232.

Ganchrow JR and Erickson RP. Neural correlates of gustatory intensity and quality. J Neurophysiol 33: 768-783, 1970.

Halpern BP. Time as a factor in gustation: temporal patterns of stimulation and response. In: Taste, Olfaction, and the Central Nervous System, edited by Pfaff DW. New York: Rockefeller University Press, 1985, p. 181-209.

Halpern BP and Tapper DN. Taste stimuli: quality coding time. Science 171: 1256-1258, 1971.

Halsell CB, Travers SP, and Travers JB. Ascending and descending projections from the rostral nucleus of the solitary tract originate from separate neuronal populations. Neuroscience 72: 185-197, 1996.

Hopfield JJ. Pattern recognition computation using action potential timing for stimulus representation. Nature 376: 33-36, 1995.

Katz DB, Nicolelis MA, and Simon SA. Gustatory processing is dynamic and distributed. Curr Opin Neurobiol 12: 448-454, 2002.

Katz DB, Simon SA, and Nicolelis MA. Dynamic and multimodal responses of gustatory cortical neurons in awake rats. J Neurosci 21: 4478-4489, 2001 .

König P, Engel AK, and Singer W. Integrator or coincidence detector? The role of the cortical neuron revisited. Trends Neurosci 19: 130-137, 1996.

Laurent G. A systems perspective on early olfactory coding. Science 286: 723-728, 1999.

Lundy RF and Contreras RJ. Gustatory neuron types in rat geniculate ganglion. J Neurophysiol 82: 2970-2988, 1999.
McClurkin JW, Optican LM, Richmond BJ, and Gawne TJ. Concurrent processing and complexity of temporally encoded neuronal messages in visual perception. Science 253: 675-677, 1991.

Mistretta CM. A quantitative analysis of rat chorda tympani fiber discharge patterns. In: Olfaction and Taste IV, edited by Schneider D. Stuttgart, Germany: Wissenschaftliche Verlagsgesellschaft, 1972, p. 294-300.

Nagai T and Ueda K. Stochastic properties of gustatory impulse discharges in rat chorda tympani fibers. J Neurophysiol 45: 574-592, 1981.

Nishijo $\mathbf{H}$ and Norgren R. Parabrachial gustatory neural activity during licking by rats. $J$ Neurophysiol 66: 974-985, 1991.

Nuding SC, McPheeters M, and Frank ME. Interspike interval patterns of taste neurons in the hamster solitary nucleus. Chem Senses 16: 429-446, 1991

Ogawa H, Sato M, and Yamashita S. Variability in impulse discharges in rat chorda tympani fibers in response to repeated gustatory stimulation. Physiol Behav 11: 469-479, 1973.

Ogawa H, Yamashita S, and Sato M. Variation in gustatory nerve fiber discharge pattern with change in stimulus concentration and quality. $J \mathrm{Neu}$ rophysiol 37: 443-447, 1974.

Reich DS, Mechler F, and Victor JD. Temporal coding of contrast in primary visual cortex: when, what, and why? J Neurophysiol 85: 1039-1050, 2001.

Ringach DL, Hawken MJ, and Shapley R. Dynamics of orientation tuning in macaque primary visual cortex. Nature 387: 281-284, 1997.

Scott TR. Behavioral support for a neural taste theory. Physiol Behav 12: 413-417, 1974.

Scott TR and Erickson RP. Synaptic processing of taste-quality information in thalamus of the rat. J Neurophysiol 34: 868-884, 1971.

Scott TR and Giza BK. Coding channels in the taste system of the rat. Science 249: 1585-1587, 1990.

Scott TR and Mark GP. Feeding and taste. Prog Neurobiol 27: 293-317, 1986.

Scott TR and Perrotto RS. Intensity coding in pontine taste area: Gustatory information is processed similarly throughout rat's brain stem. J Neurophysiol 44: 739-750, 1980.

Shapley R and Enroth-Cugell C. Visual adaptation and retinal gain controls. Prog Retinal Res 3: 263-346, 1984.

Smith DV and Travers JB. A metric for the breadth of tuning of gustatory neurons. Chem Senses Flav 4: 215-229, 1979.

Softky W. Sub-millisecond coincidence detection in active dendritic trees. Neuroscience 58: 13-41, 1994.

Reich DS, Mechler F, and Victor JD. Temporal coding of contrast in primary visual cortex: when, what, and why? J Neurophysiol 85: 1039-1050, 2001.

Travers SP and Hu H. Extranuclear projections of rNST neurons expressing gustatory-elicited Fos. J Comp Neurol 427: 124-138, 2000.

Travers SP and Norgren R. The time course of solitary nucleus gustatory response: influence of stimulus and site of application. Chem Senses 14: 55-74, 1989.

Verhagen JV, Giza BK, and Scott TR. Responses to taste stimulation in the ventroposteromedial nucleus of the thalamus in rats. J Neurophysiol 89: 265-275, 2003.

Victor JD. Temporal aspects of neural coding in the retina and lateral geniculate. Network 10: R1-66, 1999.

Victor JD and Purpura KP. Nature and precision of temporal coding in visual cortex: a metric-space analysis. J Neurophysiol 76: 1310-1326, 1996.

Victor JD and Purpura KP. Metric-space analysis of spike trains: theory, algorithms and application. Network 8: 127-164, 1997.

Yamamoto $\mathbf{T}$ and Yuyama N. On a neural mechanism for cortical processing of taste quality in the rat. Brain Res 400: 312-320, 1987.

Yamamoto T, Yuyama N, Kato T, and Kawamura Y. Gustatory responses of cortical neurons in rats. I. Response characteristics. J Neurophysiol 51: 615-635, 1984. 